\title{
Appendix 1: Search strategies
}

\section{RAPID RESPONSE SCHEMES}

The search strategies were modified versions of those used by NICE in CG50 (2007). The searches were run on 21 October 2014 in Embase and MEDLINE, and limited to records added to the databases from December 2006 onwards.

\section{Medline}

1. $\exp$ Critical care/

2. Critical care\$.tw.

3. exp *Intensive Care Units/

4. intensive care\$.tw.

5. ((critical\$ or acute\$ or sever\$ or sudden\$ or unexpected\$) adj2 ill\$).tw.

6. (patient\$ adj2 deterior\$).tw.

7. (risk\$ adj2 deterior\$).tw.

8. critical illness/

9. (clinical\$ adj2 deterior\$).tw.

10. Heart Arrest/ep, mo, pc [Epidemiology, Mortality, Prevention \& Control]

11.1 or 2 or 3 or 4 or 5 or 6 or 7 or 8 or 9 or 10

12. exp patient care team/

13. outreach.tw.

14. patient at risk\$.tw.

15. patient care team\$.tw.

16. hospital emergency team\$.tw.

17. 12 or 13 or 14 or 15 or 16

18. 11 and 17

19. rapid response team\$.tw.

20. medical emergency team\$.tw.

21. Hospital Rapid Response Team/

22. rapid response system\$.tw.

23. (outreach adj (service\$ or team\$)).tw.

24. 19 or 20 or 21 or 22 or 23

25. 18 or 24

26. $200612 \$$.ed.

27. $2007 \$ . e d$. 
28. 2008\$.ed.

29. 2009\$.ed.

30. 2010\$.ed.

31. 2011\$.ed.

32. $2012 \$$.ed

33. $2013 \$$.ed.

34. 2014 \$.ed.

35. or/26-34

36. 25 and 35

37. limit 36 to english language

\section{EARLY WARNING SCORES}

The search strategies were modified versions of those used by NICE in CG50 (2007). The searches were run on 21 October 2014 in Embase and MEDLINE and limited to records added to the databases from 31 October 2006 onwards.

\section{Medline}

1. *Health Status Indicators/

2. exp * Severity of Illness Index/

3. *Risk Assessment/

4. severity of illness ind\$.tw.

5. health status ind\$.tw.

6. risk assess\$.tw.

7. early warning.tw.

8. (warning adj2 (scor\$ or system\$)).tw.

9. (track and trigger).tw.

10. ((trigger or calling) adj5 criteria).tw.

11. *Point-of-care Systems/

12. point of care system\$.tw.

13. serious\$ ills.tw.

14. or/1-13

15. exp *Critical Care/

16. critical care.tw.

17. intensive care.tw. 
18. exp *Intensive Care Units/

19. Hospital Rapid Response Team/

20. rapid response system\$.tw.

21. rapid response team\$.tw.

22. medical emergency team\$.tw.

23. hospital emergency team\$.tw.

24. exp *Patient Care team/

25. patient care team\$.tw.

26. patient at risk\$.tw.

27. (outreach adj (service\$ or team\$)).tw.

28. shock team\$.tw.

29. *critical illness/

30. ((critical\$ or acute\$ or sever\$ or sudden\$ or unexpected\$) adj2 ill\$).tw.

31. (patient\$ adj2 deterior\$).tw.

32. (risk\$ adj2 deterior\$).tw.

33. Heart arrest/ep, mo, pc

34. or/15-33

35. 14 and 34

36. 200611\$.ed.

37. $200612 \$$.ed.

38. 2007 .ed.

39. 2008 \$.ed.

40. 2009\$.ed.

41. 2010\$.ed.

42. 2011\$.ed.

43. $2012 \$$.ed.

44. 2013\$.ed.

45. 2014\$.ed.

46. or/36-45

47. 35 and 46

48. limit 47 to english language 


\section{STANDARDISED HANDOVER TOOLS}

\section{Search strategy}

The search strategies were modified versions of those used by Robertson et al (2014). The searches were run on 21 October 2014 and limited to records added to the databases from July 2012 onwards.

\section{Medline}

1. patient handoff/

2. handover?.tw.

3. hand-over?.tw.

4. handoff?.tw.

5. hand-off?.tw.

6. signout?.tw.

7. sign-out?.tw.

8. patient transfer/

9. patient transfer\$.tw.

10. intrahospital transfer\$.tw.

11. intra-hospital transfer\$.tw.

12. intrahospital transport\$s.tw.

13. intra-hospital transport\$.tw.

14. shift to shift.tw.

15. intershift.tw.

16. inter-shift.tw.

17. or/1-16

18. quality improvement/

19. intervention*.tw.

20. (improv* and quality).tw.

21. (improv* and safety).tw.

22. strateg*.tw.

23. tool\$.tw.

24. training.tw.

25. instrument\$.tw.

26. standardi*.tw.

27. mneumonic\$.tw. 
28. or/18-27

29. 17 and 28

30. SBAR.tw.

31. ISBAR.tw.

32. 30 or 31

33. 29 or 32

34. 201207\$.ed.

35. 201208\$.ed.

36. $201209 \$$.ed.

37. 201210\$.ed.

38. 201211\$.ed.

39. $201212 \$$.ed.

40. $2013 \$$.ed.

41. 2014\$.ed.

42. or/34-41

43. 33 and 42

44. limit 43 to english language

\section{CONTINUING EDUCATION}

The searches were run on 21 October 2014 and limited to records added to the databases from 1990 onwards.

\section{Medline}

1. exp Critical care/

2. Critical care\$.tw.

3. ((critical\$ or acute\$ or sever\$ or sudden\$ or unexpected\$) adj2 ill\$).tw.

4. (patient\$ adj2 deterior\$).tw.

5. (risk\$ adj2 deterior\$).tw.

6. (clinical\$ adj2 deterior\$).tw.

7. critical illness/

8. or/1-7

9. *education, continuing/ or *education, medical, continuing/ or *education, nursing, continuing/ or *education, professional, retraining/ or *education, medical/ or *education, nursing/

10. medical education.tw. 
11. nurs\$ education.tw.

12. exp *teaching/

13. exp *inservice training/

14. or/9-13

15. immediate life support\$.tw.

16. Life Support Care/ed

17. Advanced Cardiac Life Support/ed

18. or/15-17

19. Heart arrest/ep, mo, pc

20. 8 or 19

21. 20 and 14

22. 21 or 18

23. limit 22 to $y r=" 1990$-Current"

24. limit 23 to english language 
Appendix 2: High quality review papers used as foundation for the search strategy

\begin{tabular}{|l|l|l|l|l|}
\hline Topic & $\begin{array}{l}\text { Original systematic } \\
\text { review }\end{array}$ & $\begin{array}{l}\text { Original search } \\
\text { start/end }\end{array}$ & $\begin{array}{l}\text { Our search start } \\
\text { date }\end{array}$ & $\begin{array}{l}\text { Our search end } \\
\text { date }\end{array}$ \\
\hline $\begin{array}{l}\text { Rapid response } \\
\text { schemes }\end{array}$ & $\begin{array}{l}\text { NICE Clinical } \\
\text { Guideline 50 }\end{array}$ & $\begin{array}{l}\text { Jan 2004-Dec } \\
2006\end{array}$ & December 2006 & October 21 2014 \\
\hline $\begin{array}{l}\text { Early warning } \\
\text { scores }\end{array}$ & $\begin{array}{l}\text { NICE Clinical } \\
\text { Guideline 50 }\end{array}$ & $\begin{array}{l}\text { Nov 2004- } \\
\text { October 2006 }\end{array}$ & October 2006 & October 21 2014 \\
\hline $\begin{array}{l}\text { Standardised } \\
\text { handover tool }\end{array}$ & $\begin{array}{l}\text { Robertson et al } \\
2014\end{array}$ & $\begin{array}{l}\text { January 2002- } \\
\text { July 2012 }\end{array}$ & July 2012 & October 21 2014 \\
\hline $\begin{array}{l}\text { Continuing } \\
\text { education }\end{array}$ & None found & & January 1990 & October 21 2014 \\
\hline
\end{tabular}




\begin{tabular}{|c|c|}
\hline $1++$ & $\begin{array}{l}\text { High-quality meta-analyses, systematic reviews of RCTs, or RCTs with a very low risk } \\
\text { of bias }\end{array}$ \\
\hline $1+$ & $\begin{array}{l}\text { Well-conducted meta-analyses, systematic reviews of RCTs, or RCTs with a low risk } \\
\text { of bias }\end{array}$ \\
\hline $1-$ & Meta-analyses, systematic reviews of RCTs, or RCTs with a high risk of bias \\
\hline $2++$ & $\begin{array}{l}\text { High-quality systematic reviews of case-control or cohort studies } \\
\text { High-quality case-control or cohort studies with a very low risk of confounding, bias } \\
\text { or chance and a high probability that the relationship is causal }\end{array}$ \\
\hline $2+$ & $\begin{array}{l}\text { Well-conducted case-control or cohort studies with a low risk of confounding, bias } \\
\text { or chance and a moderate probability that the relationship is causal }\end{array}$ \\
\hline 2- & $\begin{array}{l}\text { Case-control or cohort studies with a high risk of confounding, bias, or chance and a } \\
\text { significant risk that the relationship is not causal }\end{array}$ \\
\hline 3 & Non-analytic studies (for example, case reports, case series) \\
\hline 4 & Expert opinion, formal consensus \\
\hline
\end{tabular}




\section{Appendix 4: Data items extracted from papers}

Study Type

- Study design

- Data collection method

- Study duration (observation, intervention and follow-up)

Population

- Number of participants

- $\quad$ Setting

- Age

- Gender

- Inclusion and exclusion criteria

Risk of bias

- Selection bias

- Performance bias

- Attrition bias

- Detection bias

- Other concerns about bias

Intervention type

- Aim, intervention and control details

Outcomes

Funding source 
Appendix 5: Exclusion criteria

\begin{tabular}{|c|c|c|c|}
\hline $\begin{array}{l}\text { Rapid response } \\
\text { schemes }\end{array}$ & $\begin{array}{l}\text { Early warning } \\
\text { scores }\end{array}$ & $\begin{array}{l}\text { Standardised } \\
\text { handover tools }\end{array}$ & $\begin{array}{l}\text { Continuing } \\
\text { education }\end{array}$ \\
\hline $\begin{array}{l}50 \% \text { or more of } \\
\text { patients included } \\
\text { were: under 18; } \\
\text { dying patients } \\
\text { receiving } \\
\text { palliative care; not } \\
\text { on general adult } \\
\text { wards (e.g. } \\
\text { primary care, CCU, } \\
\text { ICU, A\&E, } \\
\text { catheterization } \\
\text { labs, theatre). } \\
\text { reviews }\end{array}$ & $\begin{array}{l}50 \% \text { or more } \\
\text { of patients } \\
\text { included were: } \\
\text { under 18; dying } \\
\text { patients } \\
\text { receiving } \\
\text { palliative care; } \\
\text { not on general } \\
\text { adult wards (e.g. } \\
\text { primary care, } \\
\text { CCU, ICU, A\&E, } \\
\text { catheterization } \\
\text { labs, theatre). } \\
\text { Non- } \\
\text { systematic } \\
\text { reviews } \\
\text { Limited to } \\
\text { single parameter } \\
\text { systems }\end{array}$ & $\begin{array}{l}\text { Handover } \\
\text { setting focused } \\
\text { outside of } \\
\text { general adult } \\
\text { wards (e.g. } \\
\text { primary care, } \\
\text { paediatric, } \\
\text { mental health, } \\
\text { CCU, ICU, A\&E, } \\
\text { catheterization } \\
\text { labs, theatre). } \\
\text { Non- } \\
\text { systematic } \\
\text { reviews }\end{array}$ & $\begin{array}{l}\text { Fewer than } 50 \% \\
\text { of the subjects were } \\
\text { practicing doctors or } \\
\text { nurses working on } \\
\text { adult general in- } \\
\text { patient wards. } \\
\text { Intervention } \\
\text { targets continuing } \\
\text { medical education in } \\
\text { a specialty specific } \\
\text { context (e.g. } \\
\text { paediatrics or critical } \\
\text { care). } \\
\text { Intervention } \\
\text { focused on teaching } \\
\text { response to full } \\
\text { arrest scenarios } \\
\text { Asked participants } \\
\text { after the } \\
\text { intervention, to state } \\
\text { how they thought } \\
\text { their pre/post } \\
\text { intervention } \\
\text { knowledge } \\
\text { compared. }\end{array}$ \\
\hline
\end{tabular}


Appendix 6: Population, Intervention, Comparison, Outcome

\begin{tabular}{|c|c|c|c|c|}
\hline Topic & Population & Intervention & Comparison & Outcome \\
\hline $\begin{array}{l}\text { Rapid } \\
\text { response } \\
\text { schemes }\end{array}$ & $\begin{array}{l}\text { Adult in- } \\
\text { patients on } \\
\text { general } \\
\text { medical or } \\
\text { surgical wards. }\end{array}$ & $\begin{array}{l}\text { Introduction of a } \\
\text { rapid response scheme } \\
\text { (team that responds to } \\
\text { calls for help managing } \\
\text { deteriorating patients). }\end{array}$ & $\begin{array}{l}\text { Current or } \\
\text { historic } \\
\text { comparison } \\
\text { group. }\end{array}$ & $\begin{array}{l}\text { Any that } \\
\text { evaluate } \\
\text { effectiveness of } \\
\text { the intervention. }\end{array}$ \\
\hline $\begin{array}{l}\text { Early } \\
\text { warning } \\
\text { scores }\end{array}$ & $\begin{array}{l}\text { Adult in- } \\
\text { patients on } \\
\text { general } \\
\text { medical or } \\
\text { surgical wards. }\end{array}$ & $\begin{array}{l}\text { Introduction of a } \\
\text { track and trigger } \\
\text { system (recording of } \\
\text { patient observations } \\
\text { with a defined } \\
\text { threshold which } \\
\text { triggers a response). }\end{array}$ & $\begin{array}{l}\text { Current or } \\
\text { historic } \\
\text { comparison } \\
\text { group. }\end{array}$ & $\begin{array}{l}\text { Any that } \\
\text { evaluate } \\
\text { effectiveness of } \\
\text { the intervention. }\end{array}$ \\
\hline $\begin{array}{l}\text { Standardised } \\
\text { handover } \\
\text { tool }\end{array}$ & $\begin{array}{l}\text { Qualified } \\
\text { doctors and } \\
\text { nurses working } \\
\text { on adult } \\
\text { general } \\
\text { medical or } \\
\text { surgical wards } \\
\text { in hospitals. }\end{array}$ & $\begin{array}{l}\text { Introduction of a } \\
\text { standardised tool to } \\
\text { structure } \\
\text { communication during } \\
\text { intra-hospital } \\
\text { handover of patient } \\
\text { information e.g. } \\
\text { standardised handover } \\
\text { sheets. }\end{array}$ & $\begin{array}{l}\text { Current or } \\
\text { historic } \\
\text { comparison } \\
\text { group. }\end{array}$ & $\begin{array}{l}\text { Any that } \\
\text { evaluate } \\
\text { effectiveness of } \\
\text { the intervention. }\end{array}$ \\
\hline $\begin{array}{l}\text { Continuing } \\
\text { education }\end{array}$ & $\begin{array}{l}\text { Qualified } \\
\text { doctors and } \\
\text { nurses working } \\
\text { on adult } \\
\text { general } \\
\text { medical or } \\
\text { surgical wards } \\
\text { in hospitals. }\end{array}$ & $\begin{array}{l}\text { Implementation of } \\
\text { an educational } \\
\text { intervention aimed at } \\
\text { improving the subject's } \\
\text { identification and } \\
\text { management of } \\
\text { deteriorating adult } \\
\text { inpatients not being } \\
\text { managed in critical } \\
\text { care areas. }\end{array}$ & $\begin{array}{l}\text { Current or } \\
\text { historic } \\
\text { comparison } \\
\text { group. }\end{array}$ & $\begin{array}{l}\text { Any that } \\
\text { evaluate } \\
\text { effectiveness of } \\
\text { the intervention. }\end{array}$ \\
\hline
\end{tabular}




\section{Appendix 7: PRISMA flow diagrams}

\section{Rapid response schemes}
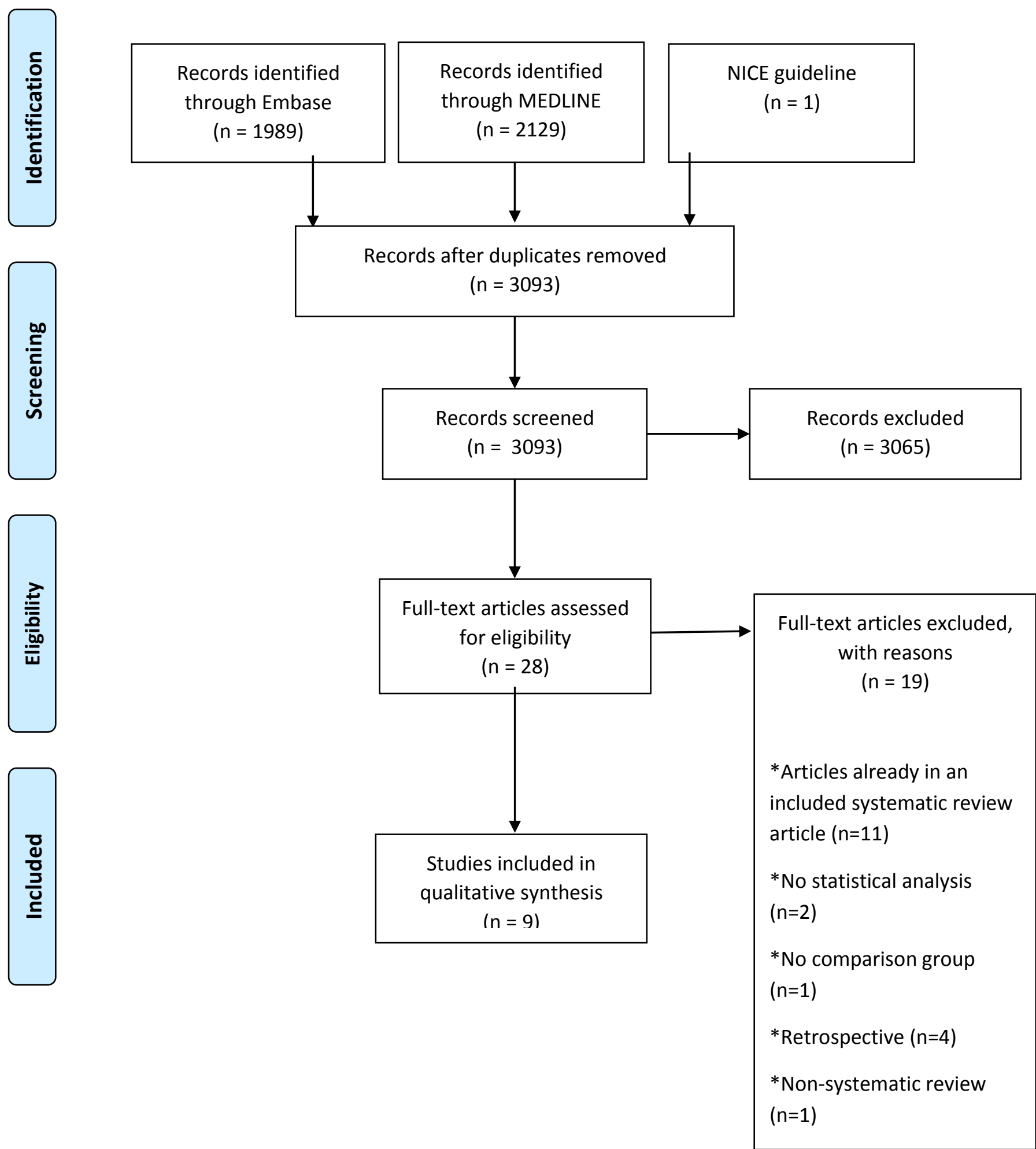


\section{Early warning scores}
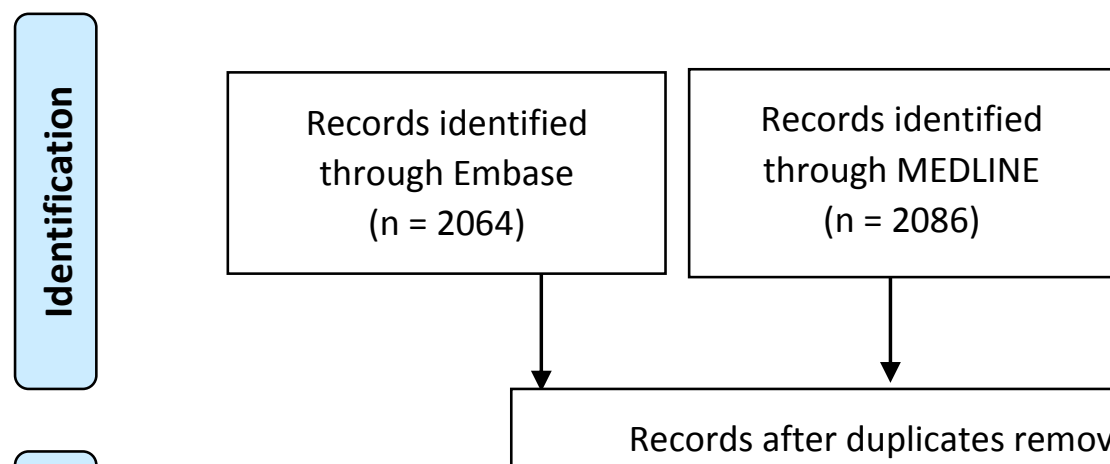

Records after duplicates removed

$(n=3267)$
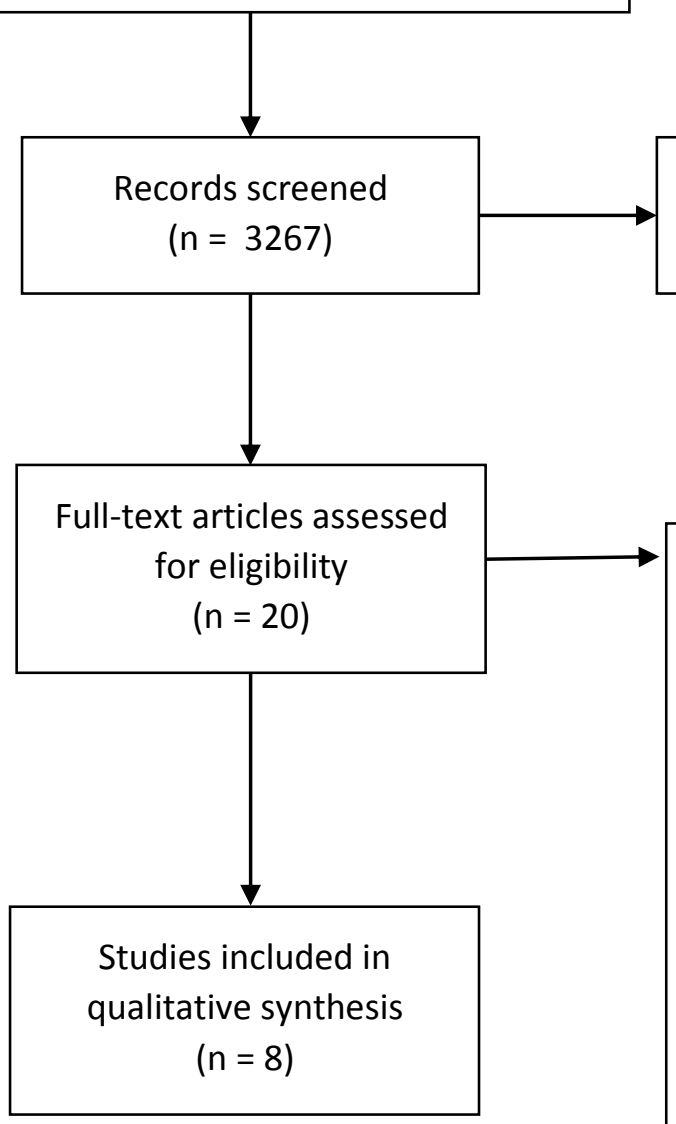

Records excluded

$(n=3247)$

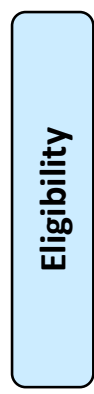

Full-text articles excluded, with reasons

$(n=12)$

*Validation not

implementation $(n=2)$

웜

*Descriptive $(\mathrm{n}=2)$

*Abstract only $(n=1)$

*Non-systematic review $(n=3)$

*Retrospective audit ( $\mathrm{n}=1)$

*Single parameter system $(n=1)$

* Included in existing systematic review $(n=2)$ 
Standardised handover tools

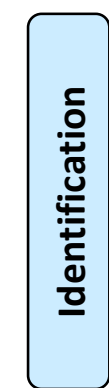

Records identified through Embase

$$
\text { ( } n=1689 \text { ) }
$$
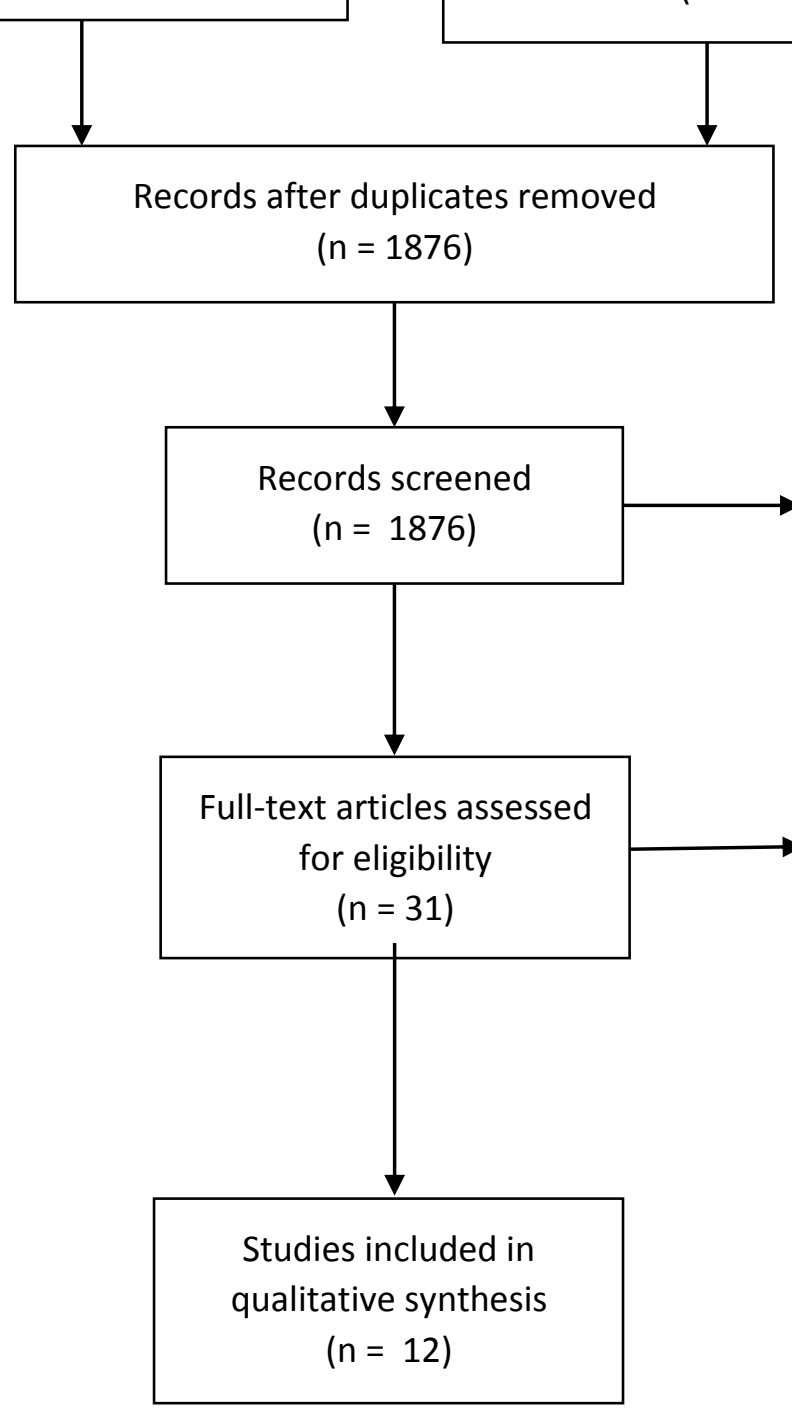

Records excluded $(n=1845)$
Full-text articles excluded, with reasons

$$
(n=19)
$$

*Handover not on ward $(n=3)$

*No statistical tests $(n=1)$

*Sample size unclear $(n=1)$

*No control group ( $n=2)$

*Non-systematic review $(n=6)$

*Descriptive only $(n=1)$

*Paediatrics focus $(n=1)$

*Handover minor $(n=1)$

*No intervention $(n=2)$

*Retrospective $(n=1)$ 
Continuing education

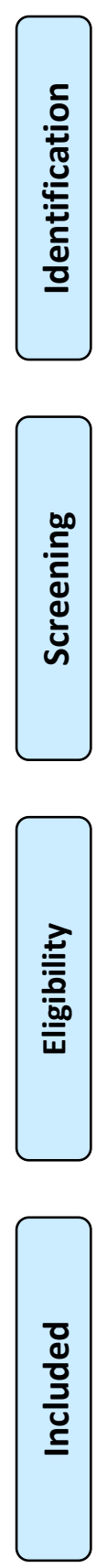

Records identified through Embase

$(n=1470)$
Records identified through MEDLINE

( $n=1475)$
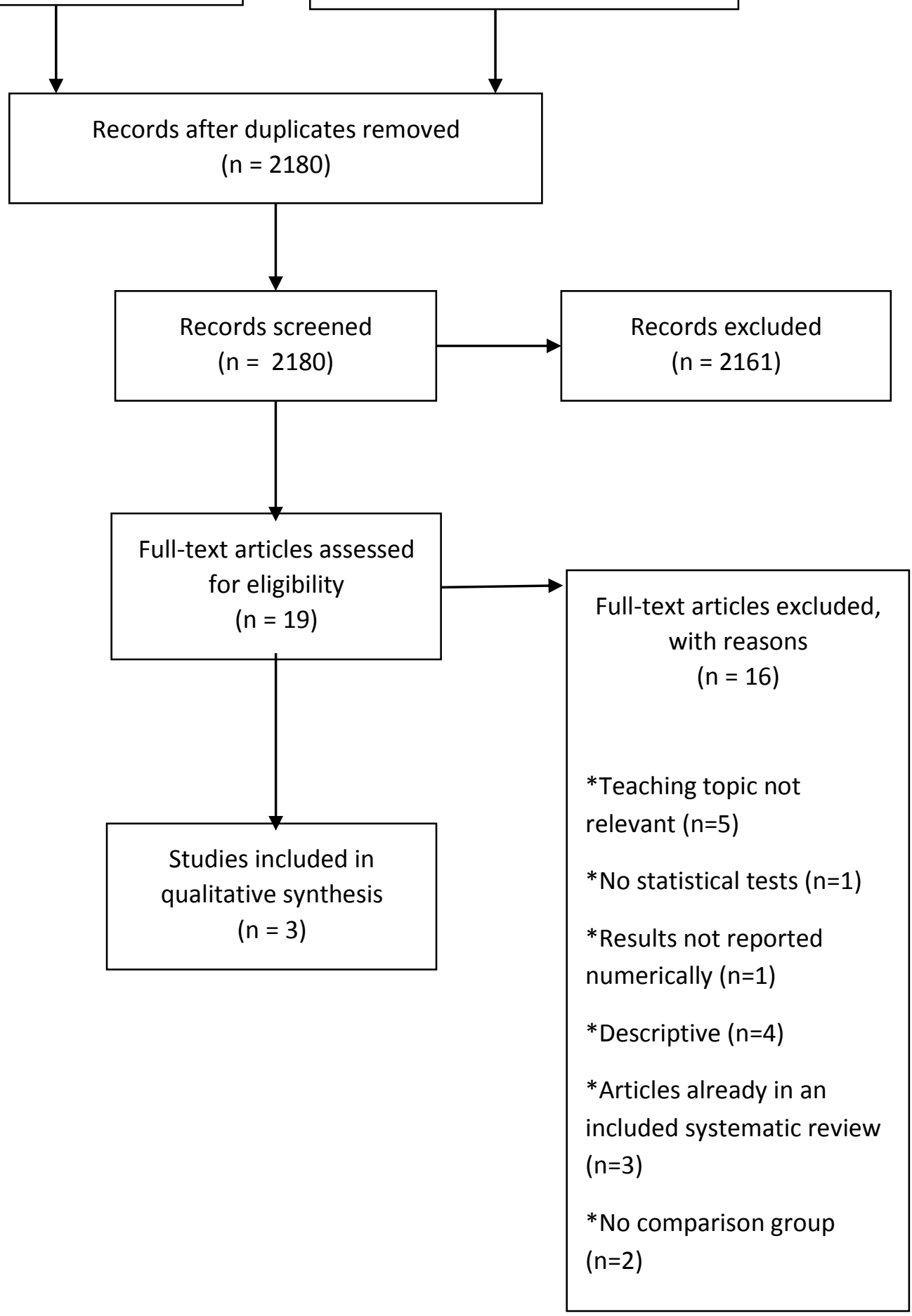